\title{
A SPECTRAL THEOREM FOR NONLINEAR OPERATORS
}

\author{
BY WILLIAM ARVESON ${ }^{1}$
}

Communicated by Paul R. Halmos, January 24, 1976

The most useful form of the spectral theorem asserts that every bounded normal operator on a complex Hilbert space is unitarily equivalent to a multiplication operator. In this note we report a (verbatim) generalization of that theorem to nonlinear operators. While the need for this result arose in attempting to define "spectral" invariants for nonlinear random processes [1], it appears to be a basic statement in operator theory which may be applicable elsewhere.

Let $H$ and $K$ be (separable) complex Hilbert spaces. By a bounded holomorphic operator from $H$ to $K$ we mean a mapping $F: H \rightarrow K$ which satisfies

(i) $\sup _{\|z\| \leqslant 1}\|F(z)\|<\infty$,

(ii) for every $z_{1}, \ldots, z_{n}$ in $H$ and every $w \in K$,

$$
\left(F\left(a_{1} z_{1}+\cdots+a_{n} z_{n}\right), w\right)
$$

defines an entire function of the $n$ complex variables $a_{1}, \ldots, a_{n}$.

Every linear operator is of course holomorphic. More generally, if $F$ is a monomial in the sense that it has the form $F(z)=G(z, z, \ldots, z)$, where $G$ is a bounded $n$-linear operator from $H \times \cdots \times H$ into $K$, then $F$ is holomorphic.

Linear spectral theory applies to operators from $H$ into itself. In the nonlinear (holomorphic) case, the appropriate range space is not $H$ but the Fock space over $\mathrm{H}$, which we will write as $e^{\mathrm{H}}$ :

$$
e^{H}=H^{0} \oplus H^{1} \oplus H^{2} \oplus \cdots,
$$

where $H^{0}=\mathbf{C}$ and $H^{n}$ is the symmetric Hilbert space tensor product of $n$ copies of $H, n \geqslant 1$. A key feature of this construction is that there is a natural representation $\pi$ of the full unitary group $U(H)$ of $H$ as unitary operators on $e^{\mathcal{H}}$. For each unitary $U$ on $H, \pi(U)$ is defined as $U^{0} \oplus U^{1} \oplus U^{2} \oplus \cdots$, where $U^{0}=1$ and $U^{n}$ is the $n$-fold tensor product of copies of $U$. This representation has been studied in some detail by Irving Segal in connection with the mathematical description of quantum systems having infinitely many degrees of freedom [2], [3]. It also plays an essential role in the following considerations.

A linear operator is normal iff it belongs to an abelian von Neumann algebra. This property can be generalized to bounded holomorphic operators $F: H$ $\rightarrow e^{H}$ as follows. We will say that $F$ commutes with a unitary $U$ in $U(H)$ if 
$F U=\pi(U) F$. If $F$ commutes with every unitary operator in the commutant of a given selfadjoint family $S$ of operators on $H$, then we say that $F$ is associated with $S$. Clearly this is the same as saying $F$ is associated with the von Neumann algebra generated by $S$. Finally, $F$ is called normal if it is associated with an abelian von Neumann algebra.

We remark that all the operators which occur naturally in the probabilistic problem alluded to in the first paragraph are normal. In that setting, there is an underlying stationary (Gaussian) random process, and the von Neumann algebra the operators are associated with is generated by the (abelian) unitary translation group of the process.

EXAMPLE: MULTIPLICATION OPERATORS. Let $(X, \mu)$ be a finite separable measure space. For each $n \geqslant 1$, let $\left(X^{n}, \mu^{n}\right)$ be the $n$-fold product measure space. If we take $H$ as $L^{2}(X, \mu)$, then there is a natural identification of $H^{n}$ with the space $L_{s}^{2}\left(X^{n}, \mu^{n}\right)$ of all symmetric functions in $L^{2}\left(X^{n}, \mu^{n}\right)$. Thus the Fock space over $L^{2}(X, \mu)$ is simply

$$
L^{2}\left(X^{0}, \mu^{0}\right) \oplus L^{2}(X, \mu) \oplus L_{s}^{2}\left(X^{2}, \mu^{2}\right) \oplus \cdots
$$

where $\left(X^{0}, \mu^{0}\right)$ is taken as the trivial one-point measure space. Now for each $n$ $\geqslant 0$, let $a_{n}=a_{n}\left(x_{1}, x_{2}, \ldots, x_{n}\right)$ be a bounded (measurable) symmetric function on $X^{n}$. Then, under appropriate mild growth conditions on the sequence of norms $\left\|a_{n}\right\|_{\infty}$, we can define a bounded holomorphic operator $F: H \rightarrow e^{\mathcal{H}}$ as the (direct) $\operatorname{sum} F(z)=F_{0}(z)+F_{1}(z)+\cdots$ where, for $z \in L^{2}(X, \mu), F_{n}(z) \in$ $L_{S}^{2}\left(X^{n}, \mu^{n}\right)$ is defined by

$$
F_{n}(z)\left(x_{1}, \ldots, x_{n}\right)=a_{n}\left(x_{1}, \ldots, x_{n}\right) z\left(x_{1}\right) z\left(x_{2}\right) \cdots z\left(x_{n}\right)
$$

It is easy enough to see that $F$ is normal, and in fact is associated with the multiplication algebra of $L^{2}(X, \mu)$, a maximal abelian von Neumann algebra. A nonlinear operator such as $F$ will be called a multiplication operator based on $(X, \mu)$.

Finally, we need an appropriate version of unitary equivalence. Two operators $F: H \rightarrow e^{\mathcal{H}}$ and $G: K \rightarrow e^{K}$ are said to be equivalent if there is a unitary operator $W$ from $H$ to $K$ such that $G W=\widetilde{W} F$ where $\widetilde{W}$ is the unitary operator from $e^{\mathcal{H}}$ to $e^{K}$ induced by $W$ in the natural way (by direct summing appropriate tensor powers of $W$ ).

The main result is,

Spectral Theorem. Let $F: H \longrightarrow e^{H}$ be a bounded holomorphic normal operator. Then $F$ is equivalent to a multiplication operator based on a finite separable measure space.

Full details behind the proof of this theorem, together with the results announced in [1], will appear in subsequent papers. 


\section{REFERENCES}

1. W. Arveson, Spectral theor, for nonlinear random processes, Proc. Conf. on Linear Operators, Index Theory, and $K$-Theory (Rome, Oct. 1975) (to appear).

(1956), 106-134. MR 17, 880.

3. The structure of a class of representations of the unitary group on a Hilbert space, Proc. Amer. Math. Soc. 8 (1957), 197-203. MR 18, 812.

DEPARTMENT OF MATHEMATICS, UNIVERSITY OF CALIFORNIA, BERKELEY, CALIFORNIA 94720 